\title{
Improving Accuracy and Power with Transfer Learning Using a Meta-analytic Database
}

\author{
Yannick Schwartz ${ }^{1,2}$, Gaël Varoquaux ${ }^{1,2}$, Christophe Pallier ${ }^{3,2}$, \\ Philippe Pinel $^{3,2}$, Jean-Baptiste Poline ${ }^{2}$, and Bertrand Thirion ${ }^{1,2}$ \\ 1 Parietal Team, INRIA Saclay-Île-de-France, Saclay, France \\ yannick.schwartz@inria.fr \\ 2 CEA, DSV, I²BM, Neurospin bât 145, 91191 Gif-Sur-Yvette, France \\ 3 INSERM, CEA, Cognitive Neuroimaging Unit, Neurospin Center, France
}

\begin{abstract}
Typical cohorts in brain imaging studies are not large enough for systematic testing of all the information contained in the images. To build testable working hypotheses, investigators thus rely on analysis of previous work, sometimes formalized in a so-called meta-analysis. In brain imaging, this approach underlies the specification of regions of interest (ROIs) that are usually selected on the basis of the coordinates of previously detected effects. In this paper, we propose to use a database of images, rather than coordinates, and frame the problem as transfer learning: learning a discriminant model on a reference task to apply it to a different but related new task. To facilitate statistical analysis of small cohorts, we use a sparse discriminant model that selects predictive voxels on the reference task and thus provides a principled procedure to define ROIs. The benefits of our approach are twofold. First it uses the reference database for prediction, i.e. to provide potential biomarkers in a clinical setting. Second it increases statistical power on the new task. We demonstrate on a set of 18 pairs of functional MRI experimental conditions that our approach gives good prediction. In addition, on a specific transfer situation involving different scanners at different locations, we show that voxel selection based on transfer learning leads to higher detection power on small cohorts.
\end{abstract}

Keywords: Meta-analysis, fMRI, multiple comparison, machine learning.

\section{Introduction}

Multi-subject or multi-condition experiments are the workhorse of bio-medical imaging research, whether it be drug development or basic research. Imaging provides a wealth of information on the biomedical problem at hand. However the typical sample size is too small to fully exploit this information. For this reason, investigators often turn to previous studies in order to formulate hypotheses and restrict the search space, i.e. select a subset of anatomical or functional structures of interest to the current study. A typical case is that of early-stage clinical trials, for which the group size is very small, but that are most often based on previous results concerning the pathology under study. However, understanding the literature is increasingly difficult and requires a systematic approach, that 
takes the form of a meta-analysis, pooling results from multiple experiments that address a set of related research hypotheses [1].

In particular, brain imaging studies heavily rely on such meta-analyses [2], as the brain is still an ill-understood and complex organ. In functional Magnetic Resonance Imaging (fMRI) studies, typical group sizes range from 10 to 20 subjects, which is not always enough to warrant the reliability of brain-wide analysis 3 . More importantly, the time that can be spent in the scanner by subjects is limited, and not all interesting experimental conditions will be acquired.For this reason, it is common practice to reduce the study to a set of regions of interest (ROIs) extracted from the literature. Investigators define these ROIs by extracting locations of peak activations from the literature [4], or from coordinate databases such as BrainMap [5]. While most of these meta-analyses are conducted on activation coordinates, the increase of data sharing opens the door to meta-analysis on full brain images which results in higher statistical power [6]. Previous statistical and modeling work on meta-analysis for fMRI has focused on better modeling of the reference database [2].

In this work, we are interested in the generalization power of meta-analyses on new data. We introduce a new meta-analysis method using a reference database of images to guide statistical analysis of a new dataset. In particular we rely on predictive models, useful to learn biomarkers, and use them to select relevant voxels in order to increase the statistical power of a new study.

\section{Methods}

Problem setting. We start from a reference database made of $l$ experiments, each comprising $n^{l}$ contrasts possibly acquired over multiple subjects. We denote the brain images by $\boldsymbol{X}^{l} \in \mathbb{R}^{n^{l}, p}$ with associated experimental condition $\boldsymbol{y}^{l} \in$ $\mathbb{R}^{n^{l}}$. Given a new experiment, denoted target, $\left(\boldsymbol{X}^{\star}, \boldsymbol{y}^{\star}\right) \in\left(\mathbb{R}^{n^{\star}, p}, \mathbb{R}^{n^{\star}}\right)$, we are interested in two problems: i) (biomarkers) can we predict $\boldsymbol{y}^{\star}$ from $\boldsymbol{X}^{\star}$ ? ii) (inference) can we test hypotheses on the links between $\boldsymbol{y}^{\star}$ and $\boldsymbol{X}^{\star}$, for instance in a linear model? These are ill-posed problems from the statistics standpoint, as $n^{\star} \ll p$. The root of the problem is the dimensionality of the data: medical images are composed of many voxels, typically $p \approx 50000$ with fMRI. This large number of descriptors limits statistical inference power due to multiple testing; a problem that appears in predictive approaches as the curse of dimensionality. Here, we use our reference database to better condition this statistical problem.

Transfer learning. The gist of our approach is to learn on some experiments of our database $\left(\boldsymbol{X}^{l}, \boldsymbol{y}^{l}\right)$ discriminative models that contain predictive information for the target experiment $\left(\boldsymbol{X}^{\star}, \boldsymbol{y}^{\star}\right)$. In machine learning, this problem is known as transfer learning [7. The underlying assumption of transfer learning is the same as that for meta-analysis: the reference database should contain some common information with the target experiment. Here we use a simple form of transfer learning: we train a linear classifier on an experiment in the database that is similar from the neuroscientific point of view to the new data, and use it to predict the labels of the new data. 
Selecting predictive features. We use a sparse linear classifier, specifically an $\ell_{1}$-penalized logistic regression. The motivation behind this choice of classifier is that it produces a sparse set of weights that can be used to select relevant voxels. In particular, under certain conditions, the classifier can recover with high probability the complete set of $k$ features in $\boldsymbol{X}$ that are predictive of $\boldsymbol{y}$ for a sample size of $n_{\text {min }}=\mathcal{O}(k \log p)$ [8]. The logarithmic dependence in $p$ is an appealing property in view of the dimensionality of medical imaging datasets.

In practical situations, it can be hard to control the errors on this feature selection, in particular as it depends on the choice of the amount of $\ell_{1}$ penalty. For this reason, Meinshausen and Bühlmann [9] introduce randomized variants of sparse estimators, that can be seen as sampling the posterior probability of selection and keeping only features that are selected frequently. In particular, they establish non-asymptotic recovery results for the randomized lasso, which consists in applying the Lasso on random subsamples of the data and rescaling of the regressors. Here, we adapt this strategy to classification as the logistic regression is locally equivalent to a weighted least square and recovery results can carry from square-loss regression to logistic regression [8].

We want to use transfer learning to perform screening of the voxels, i.e. eliminate many voxels that are not related to our target experiment. For this purpose, we need a low probability of rejecting relevant variables. Each iteration of the sparse logistic regression in the randomized logistic can select reliably only $k_{\max } \approx n / \log p$ variables. In the worst case situation, we have $k$ heavilycorrelated variables and one of them is selected at random by the sparse logistic regression at each iteration. For each of these variables, the probability of selecting it less than $s$ times during $m$ iterations of the randomized logistic is given by the cumulative distribution function of a binomial with per trial success ratio $1 / k$. If $s \leq m / k$, by Hoeffding's inequality, this probability goes to zero in $o(\exp m)$. In other words, if we impose a threshold $\tau=s / m$ on the selection frequency, we can recover a group of $k$ correlated variables for $\tau \leq 1 / k$.

Brain parcellations. Although randomization relaxes the conditions on recovery, a remaining necessary condition is that the regressors of interest, i.e. the values $\boldsymbol{x}_{i}$ across the subjects of the $k$ predictive voxels, must be weakly correlated 1 . Because of the large amount of smoothness present in medical images, in particular in group-level fMRI contrasts, these conditions cannot be satisfied. Indeed, values taken by a voxel are very similar to values taken by its neighbors. In addition, the numbers of subjects used in fMRI are often below the sample size required for good recovery. For these reasons we resort to feature agglomeration: using hierarchical clustering to merge neighboring voxels carrying similar information into parcels [10]. This strategy brings the double benefit of reducing the problem size, and thus the required sample size, and mitigating local correlation, at the expense of spatial resolution.

${ }^{1}$ Specifically, the condition for recovery with randomized lasso it is a lower bound on the conditioning of the sparse eigenvalues of the design matrix [9, theorem 2] and for sparse logistic regression the corresponding condition is a lower bound on the eigenvalues of the regressors of interest's covariance matrix [8, theorem 4]. 


\section{Experiments and Results}

\subsection{FRMI Datasets}

We use 3 studies for this meta-analysis. The first study (E1) [1] is composed of 322 subjects and was designed to assess the inter-subject variability in some language, calculation, and sensorimotor tasks. The second study (E2) is similar to the first one in terms of stimuli, but its data was acquired on 35 pairs of twinsubjects. The last study (E3) [12] characterizes brain regions in charge of the syntactic and the semantic processing for the language. It was performed with 40 subjects, 20 of which were stimulated by pseudowords (jabberwocky stimuli) instead of actual meaningful sentences. All the studies were pre-processed and analyzed with the standard fMRI analysis software SPM5. The data used for this work are the statistical images resulting from the intra-subject analyses across the 3 studies. E1 has 34 contrasts images available, E2 56, and E3 28. The raw images were not always acquired on the same scanner. E1 has data from a $3 \mathrm{~T}$ SIEMENS Trio, and a $3 \mathrm{~T}$ Brucker scanner; E2 data were acquired on a $1.5 \mathrm{~T}$ GE Signa; and E3 images come from the same 3T SIEMENS Trio.

\subsection{Experimental Results for Prediction}

Here we are interested in the prediction problem: using transfer learning to discriminate a pair of constrasts with an estimator trained on two other contrasts.

We used 4 different approaches to learn the discriminative models. The first approach relies on the activation likelihood estimate (ALE) method [13], as this is a commonly published method for coordinate-based meta-analyses. We extract the activation positions from the contrasts maps, and then apply a Gaussian kernel. We use the preferred FWHM of $10 \mathrm{~mm}$ [14]. The other approaches directly use the contrast images. We name raw contrasts the method based on the contrasts voxels values; contrast-specific parcels the method that uses parcels from the training set: and meta-analystic parcels the method that learns parcels from the full database. We evaluate on our base of contrasts the ability to do transfer learning, i.e to learn decision rules that carry over from one situation to another. Since we must make the assumption that the reference contrasts hold common information with the contrasts of interest, we do not try out all the possible combinations, but rather manually select pairs of contrasts from a single experiment that form a meaningful classification task (e.g., computation versus reading, or Korean language versus French language). Out of all the possible combinations, we select 35 pairs of classification task, and subsequently combine them into 18 transfer pairs, on which it is reasonable to think that the transfer could occur (e.g., computation and reading in visual instructions, transfer on computation and reading in auditory instructions). We first train a linear classifier within 6 -fold cross validation test on a first set of pairs, setting the penalization amount by nested cross-validation, we call this step inline learning. We then re-use the discriminative model on a different pair of contrasts to perform the transfer learning. The 3 studies containing language related tasks, we can transfer between pairs within an experiment, and across experiments. Among the 18 
selected transfer pairs, we find that 9 can give rise to such a transfer. Since a transfer is directed, we perform it both ways, which yields once again 18 transfer pairs to test upon. The associated prediction scores from the different methods are reported in Table 1. The general observation is that ALE yields a poorer prediction performance than any other method. This is true both for the transfer and inline predictions. We also find that brain parcellations scores similar to the raw contrasts images, and closer to the inline predictions. We find that while the contrast-specific parcels and meta-analytic parcels methods do not return the same parcels, they produce very close results. We can thus use the full database to learn a single reference parcellation to perform meta-analysis.

Table 1. Prediction scores for inline and transfer learning. trans. $=$ transfer; in. $=$ inline; comp. $=$ computation, sent $=$ sentences $($ reading), jabb. $=$ jabberwocky; $\mathrm{S}=$ sentence with one word constituents, $\mathrm{L}=$ one constituent long sentence.

\begin{tabular}{|c|c|c|c|c|c|}
\hline Names & Peaks & Contrasts & Parcels & \multicolumn{2}{|c|}{ Meta parcels } \\
\hline & trans. in. & trans. in. & trans. in. & trans. & in. \\
\hline$\overline{E 1, \text { comp./sent. } \rightarrow \text { E2, comp./sent. }}$ & 0.750 .85 & 0.880 .97 & 0.830 .96 & 0.83 & 0.96 \\
\hline E2, comp./sent. $\rightarrow$ E1, comp./sent. & 0.660 .83 & 0.880 .96 & 0.850 .95 & 0.85 & 0.96 \\
\hline E3, jabb./French $(\mathrm{L}) \rightarrow$ E3, jabb./French $(\mathrm{S})$ & 0.460 .48 & $\begin{array}{ll}0.65 & 0.67\end{array}$ & 0.620 .60 & 0.67 & 0.62 \\
\hline /French $(\mathrm{S}) \rightarrow$ E3, & 0.520 .71 & 0.670 .85 & 0.710 .85 & 0.65 & 0.79 \\
\hline$(\mathrm{L}) \rightarrow E 2$ & 0.650 .46 & 0.730 .79 & 0.650 .81 & 0.76 & 0.85 \\
\hline E2, Korean/French $\rightarrow$ E3, jabb./French $(\mathrm{L})$ & 0.730 .81 & 0.790 .85 & 0.750 .81 & 0.75 & 0.75 \\
\hline
\end{tabular}

Fig. 1. Prediction performance relative to the best performing approach: inline prediction with raw contrasts images: the p-values indicate whether the associated methods are significantly poorer than the best performing method.

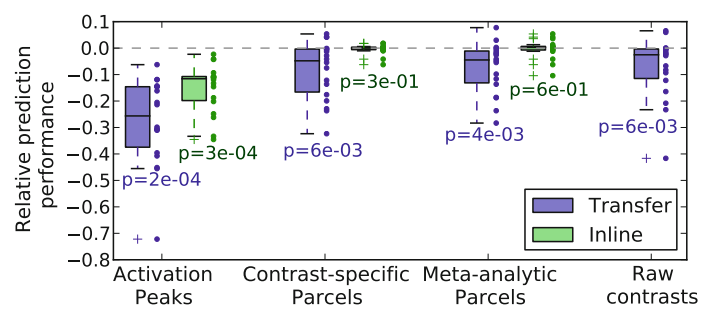

\subsection{Experimental Results for Inference}

Here we are interested in the inference problem: using transfer learning to help hypothesis testing on a target dataset. In the following, we only consider a specific transfer, namely the last line in Table 1 we learn a model discriminating French native speakers reading French or Korean, and apply it on another experiment in which French subjects had to read French or jabberwocky. This transfer is interesting as it involves two different experiments acquired on different scanners, and cognitive paradigms that share a similar expression, incomprehension of visual language stimuli. As can be seen in Table 1, the prediction scores of transfer learning as well as inline learning on this pair are acceptable although not excellent: French language and jabberwocky are difficult to separate.

Figure 2 gives the stability scores of the randomized logistic discriminating reading Korean from reading French for the different set of features -activation 
peaks, raw contrasts, parcels learned on the training contrasts or on the full database. We can see that while learning at the voxel level or at the parcel level gives similar prediction performance (Table 1), the stability score maps are very different. At the voxel-level, with 70 subjects $(p=40000, n=70)$ the recovery is limited to approximately 7 voxels without randomization: the recovery conditions are violated. As a result, the randomized logistic selects only the most predictive voxels. On the parcels, contrast-specific or meta-analytic (i.e., learned on the full database), the selection frequency highlights regions of the brain that are known to be relevant for language comprehension, including the left anterior superior temporal sulcus and the part of the temporal parietal junction (Wernicke's area).

We threshold the stability selection scores of the first experiment (Korean vs French) to select candidate voxels for the target experiment (jabberwocky vs French). As we want to perform a rough screening and would rather err on the side of false detections than false rejections, we take a very low threshold $\tau=.01$. Following our analysis above, the size of the largest group of correlated features that we can detect with such a threshold is on the order of $1 / \tau \approx 100$. With 2000 parcels, this number corresponds to $5 \%$ of the brain, i.e. 8000 voxels, and we can safely consider that no fMRI contrasts is composed of groups of heavily correlated features larger than this fraction.

On the target experiment, we perform a standard group-level analysis with the voxels selected, testing for the difference between the two conditions, jabberwocky or French reading. We report results with p-values corrected for multiple comparisons at a given family-wise error rate (FWER) using Bonferroni correction, and for a given false discovery rate (FDR) using the BenjaminiHochberg procedure. On table 2, we compare the number of detections and the detection rate, i.e. the fraction of voxels detected as significantly different, for a
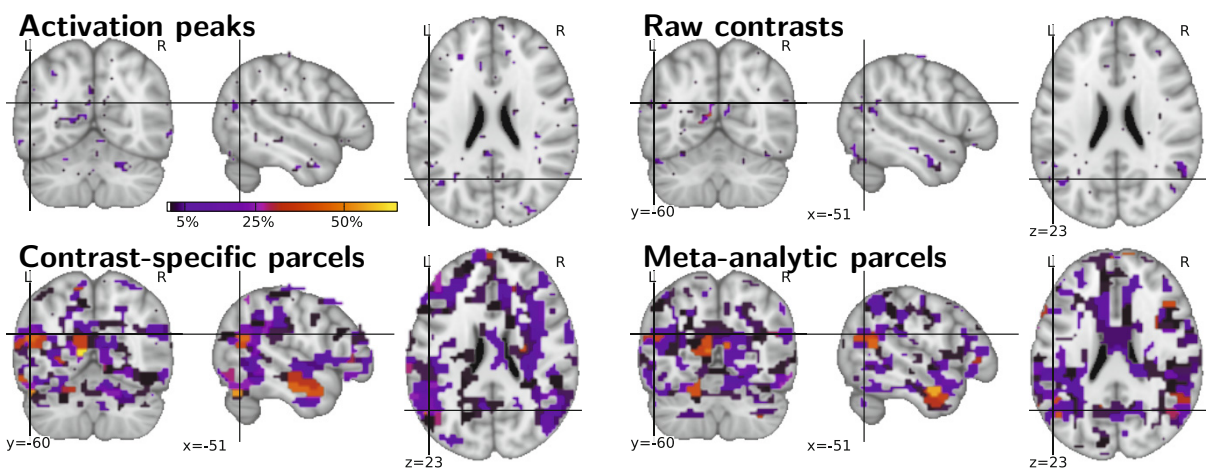

Fig. 2. Stability scores of the randomized logistic on the Korean versus French prediction of E2 for the different set of features: the colormap represents the frequency at which a feature, parcel or voxel, was selected. The maps are thresholded at $1 \%$. 

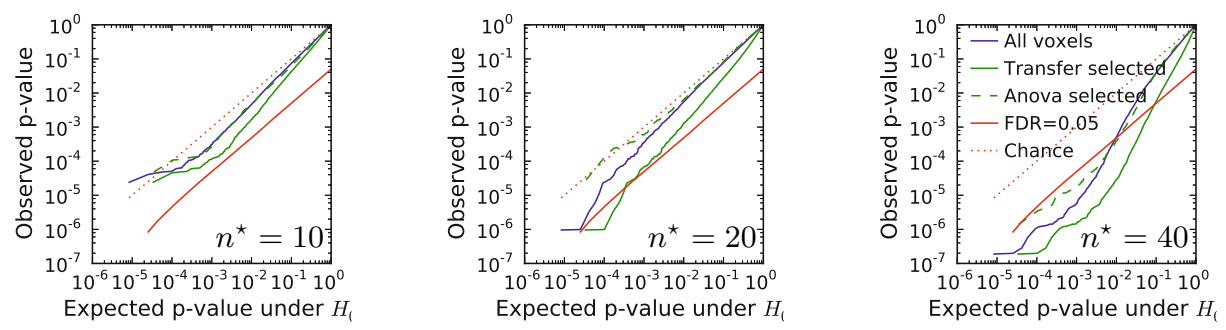

Fig. 3. Q-Q plots for the p-values with and without voxel selection by transfer learning, as well as FDR $=0.05$ threshold: left for a cohort size $n^{\star}=10$, middle for a cohort size $n^{\star}=20$, right for a cohort size $n^{\star}=40$

Table 2. Number of detections at $p<0.05$ for difference cohort size, for transfer learning and ANOVA. The percentage of detection is indicated in parenthesis.

\begin{tabular}{|c|c|c|c|c|c|c|}
\hline \multirow[b]{2}{*}{$n^{\star}$} & \multicolumn{3}{|c|}{ FWER corrected } & \multicolumn{3}{|c|}{ FDR corrected } \\
\hline & All voxels & Selection & ANOVA & All voxels & Selection & ANOVA \\
\hline$\overline{10}$ & 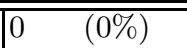 & $\overline{(\overline{(0 \%)}}$ & $\overline{\overline{(0)}}$ & $\overline{(0 \%)}$ & 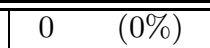 & $\overline{\overline{(0 \%)}}$ \\
\hline 20 & $(0 \%)$ & $3 \quad(0.02 \%)$ & $(0 \%)$ & $(0 \%)$ & $4 \quad(0.027 \%)$ & $(0 \%)$ \\
\hline 40 & $5 \quad(0.0084 \%)$ & $33(0.22 \%)$ & $2(0.0014 \%)$ & $143(0.97 \%)$ & $(9 \%)$ & $201(1.4 \%)$ \\
\hline
\end{tabular}

full brain analysis and for an analysis limited to the voxel selection. We compare our voxel selection method to a one-way ANOVA, and find that transfer learning outperforms the ANOVA for all the cohort sizes. Figure 3 shows the Q-Q plots on which the Benjamini-Hochberg procedure is applied. We find that voxel selection by transfer learning improves both the absolute number of detections and the detection rate for FWER and FDR correction.

\section{Conclusion}

In this paper, we propose to improve the conditioning and power of statistical analyses in imaging studies, using a large meta-analytic database.

In a transfer learning scheme, we train on the database sparse discriminative models that are suited to the target experiment. Not only can the predictive power of these models can be useful to establish biomarkers, but also they perform feature selection that can increase the statistical power of a standard group analysis on new experiments, provided enough predictive features (voxels) can be recovered. Using brain parcellations, the discriminative model acts to screen parcels unlikely to be relevant in the target experiment, thus defining automatically ROIs.

Using a set of $3 \mathrm{fMRI}$ studies related to language, we confirm experimentally that our transfer learning scheme is able to: i) perform accurate predictions on experiments acquired on a different scanner and with varying paradigm, ii) outperform the standard meta-analysis procedures based activation peaks, iii) 
increase the statistical power in the target experiment by using the ROIs defined by the discriminative model.

In this work we manually select the contrast pairs since it is delicate to interpret a transfer learning score without good knowledge of the cognitive or clinical conditions under study. Future work will study automatic contrast pairs selection, e.g. by mining the descriptions of the experiments [4, to address the problem of synthesizing the ever-growing literature and data in medical research.

\section{References}

1. Sutton, A., Abrams, K., Jones, D., Sheldon, T., Song, F.: Methods for meta-analysis in medical research, West Sussex, UK, Chichester, England (2000)

2. Wager, T.D., Lindquist, M.A., Nichols, T.E., Kober, H., Snellenberg, J.X.V.: Evaluating the consistency and specificity of neuroimaging data using meta-analysis. Neuroimage 45(suppl. 1), 210-221 (2009)

3. Thirion, B., Pinel, P., Mériaux, S., Roche, A., Dehaene, S., Poline, J.B.: Analysis of a large fmri cohort: Statistical and methodological issues for group analyses. Neuroimage 35(1), 105-120 (2007)

4. Yarkoni, T., Poldrack, R.A., Nichols, T.E., Essen, D.C.V., Wager, T.D.: Large-scale automated synthesis of human functional neuroimaging data. Nat. Methods 8(8), 665-670 (2011)

5. Laird, A.R., Lancaster, J.L., Fox, P.T.: Brainmap: the social evolution of a human brain mapping database. Neuroinformatics 3(1), 65-78 (2005)

6. Salimi-Khorshidi, G., Smith, S.M., Keltner, J.R., Wager, T.D., Nichols, T.E.: Metaanalysis of neuroimaging data: a comparison of image-based and coordinate-based pooling of studies. Neuroimage 45(3), 810-823 (2009)

7. Pan, S., Yang, Q.: A survey on transfer learning. IEEE Transactions on Knowledge and Data Engineering 22(10), 1345-1359 (2010)

8. Bach, F.: Self-concordant analysis for logistic regression. Electronic Journal of Statistics 4, 384-414 (2010)

9. Meinshausen, N., Bühlmann, P.: Stability selection. Journal of the Royal Statistical Society: Series B (Statistical Methodology) 72(4), 417-473 (2010)

10. Michel, V., Gramfort, A., Varoquaux, G., Eger, E., Keribin, C., Thirion, B.: A supervised clustering approach for fmri-based inference of brain states. Pattern Recognition (2011)

11. Pinel, P., Thirion, B., Meriaux, S., Jobert, A., Serres, J., Bihan, D.L., Poline, J.B., Dehaene, S.: Fast reproducible identification and large-scale databasing of individual functional cognitive networks. BMC Neurosci. 8, 91 (2007)

12. Pallier, C., Devauchelle, A.D., Dehaene, S.: Cortical representation of the constituent structure of sentences. Proc. Natl. Acad. Sci. U S A 108(6), 2522-2527 (2011)

13. Laird, A.R., Fox, P.M., Price, C.J., Glahn, D.C., Uecker, A.M., Lancaster, J.L., Turkeltaub, P.E., Kochunov, P., Fox, P.T.: Ale meta-analysis: controlling the false discovery rate and performing statistical contrasts. Hum. Brain Mapp. 25(1), $155-164(2005)$

14. Turkeltaub, P.E., Eden, G.F., Jones, K.M., Zeffiro, T.A.: Meta-analysis of the functional neuroanatomy of single-word reading: method and validation. Neuroimage 16(3 Pt 1), 765-780 (2002) 